\title{
IS PRIVACY INSPIRED BY WELLBEING? A PRELIMINARY STUDY
}

\author{
Ana Ferreira $^{1,2}$, Cristina Jácome ${ }^{1,2}$, Rute Almeida ${ }^{1,2}$, Filipa Lopes ${ }^{3}$, João A. Fonseca ${ }^{1,2,3}$ \\ and Pedro Vieira-Marques ${ }^{1,2}$ \\ ${ }^{1}$ CINTESIS - Center for Health Technology and Services Research \\ ${ }^{2}$ Departamento Medicina da Comunidade, Informação e Decisão em Saúde (MEDCIDS) \\ Faculty of Medicine, University of Porto, Portugal \\ ${ }^{3}$ Medicina, Educação, I\&D e Avaliação, Lda (MEDIDA) \\ Porto, Portugal
}

\begin{abstract}
Most patients with chronic diseases aim to successfully manage their disease and treatment in everyday life. Although many mobile applications are available to help them achieving this, there is still evidence of the low adherence from chronic patients to those applications. Obstacles include poor usability, lack of resources or low IT literacy, as well as the lack of trust in the privacy and security of mobile applications. Beyond the technical measures, the perception of privacy may not be a fixed concept but influenced by factors such as context, emotions and wellbeing. The aim of this paper is to investigate how health outcomes can alter the perception of privacy and trust in the use of mhealth applications, by patients with chronic respiratory diseases. Findings show that there may be significant links between patients' characteristics, their privacy perception and the type of personal data involved. The preliminary results motivate us to further research this topic.
\end{abstract}

\section{KEYWORDS}

Privacy, Trust, Patients' Perception, Wellbeing, Mhealth Apps, Chronic Patients

\section{INTRODUCTION}

Many mobile applications are available to help patients with chronic diseases to continuously and successfully manage their condition, however, barriers such as poor usability, lack of resources or low IT literacy, as well as the lack of certification and trust in mobile applications, still exist (Kruse, 2019; Zhou, 2019; Larson, 2018; Woldeyohannes, 2017). Ultimately, these obstacles hinder adherence to health enhancing behaviours, such as regular medication adherence, which can negatively affect health outcomes. In relation to users' privacy perception, most research works on mhealth applications focus on the factors that may promote/hinder the use of mhealth and, amongst these, are privacy and trust (Saheb, 2020; Wei, 2020; Vo, 2019; Majid, 2019). Privacy is closely related with users' trust in the technology, which can improve users' adherence to treatment as well as health outcomes (Hong, 2018; Serenko, 2013), as already evidenced in the trust relationship between patients and healthcare providers (Birkhäuer, 2017; Polinski, 2014). But what about the reverse? Can health outcomes influence the perception of privacy/trust in technology? Beyond technical measures of mobile applications, the perception of privacy may not be a fixed concept, but influenced by many factors (Rooy, 2010). Is patients' wellbeing one of them? The authors could only find a work studying how the severity of a disease can influence their assessment of health information's trust (Vervier, 2018). The aim of this paper is to investigate if patient health outcomes can alter patients' perception of privacy in mhealth applications.

\section{THE MOBILE APPLICATION: INSPIRERMUNDI}

The InspirerMundi application (Figure 1) is a health serious game with various monitoring functionalities to improve inhaled medication adherence in adolescents and adults with asthma (Jácome, 2017). For ubiquitous verification of adherence, the application incorporates an image-based tool for inhalers and, via image 
processing techniques, confirms the inhaler presented to the camera and locates the dose counter. This application was developed grounded in research and in close cooperation with patients and physicians. The application has been tested for usability (Ferreira, 2019) and feasibility in 26 hospital outpatient asthma clinics (Jácome, 2018). The survey presented here was applied to patients using this mobile application (Section 3).
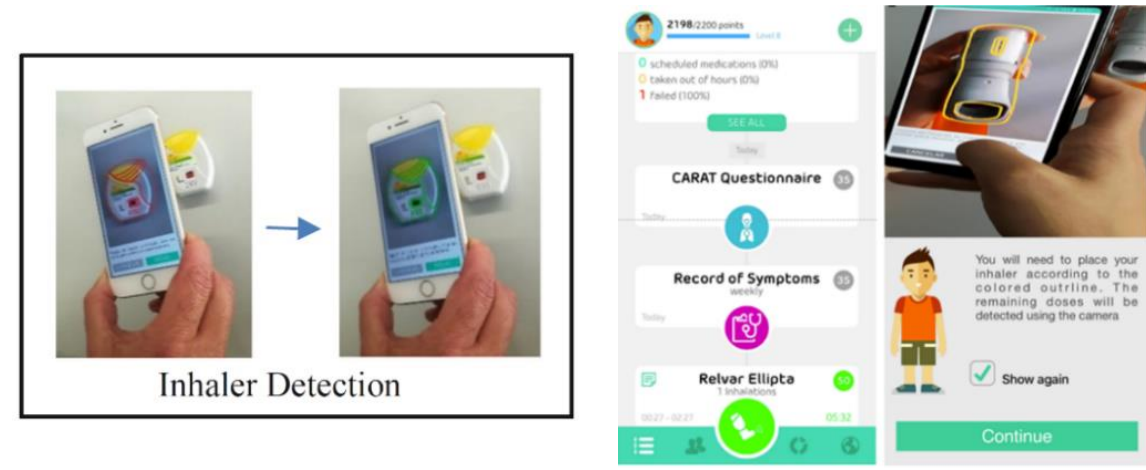

Figure 1. Example of screenshots of the InspirerMundi mobile application

\section{METHODS}

A literature review was performed with the queries presented in Table 1. Inclusion criteria comprised works from the past 5 years, written in English, which focused on the perception and attitudes towards privacy in relation to mhealth. A review of their titles and abstracts was performed and selected full papers were read and analysed. In order to search for more sources of related works, a Google query, with a combination of the terms "health, condition, influence, patient, privacy, trust", was applied, but with no further results on the subject.

For the survey, during an initial visit, patients with persistent asthma (active prescription for an inhaled controller medication) were invited by their physician to use the InspirerMundi application daily. One month later, through a telephone interview with the aim of assessing patients' feedback about the application, they were also questioned about their perception of privacy, when using mobile applications. This survey, conducted in health institutions in Portugal, included 77 participants and was approved by the ethics committees of all participating institutions and by the National Data Protection Committee (no 8825/ 2017). More details are described here (Jácome, 2018). The survey included parts such as: demographics, asthma and allergic rhinitis symptoms, medication adherence, anxiety and depression symptoms, use of social networks, mobile devices and applications, as well as four questions, evaluated with a 3 point Likert scale (never, sometimes or always), which asked if participants authorized the applications they installed, to access their: 1) Location, 2) Contacts, 3) Photographs, and allowed those applications to 4) send them Notifications. The interviews were performed between March and June 2018, and analysed in May 2020 with IBM SPSS Statistics version 26. All variables collected in the interview were tested for association/correlation with the four security questions. The a) Spearman rank test was used to calculate the correlation between categorical ordinal variables; and the b) Eta ${ }^{2}$ Coefficient test (which is the percentage of variance in the dependent variable accounted for by the independent variable) was used to determine the strength of non-linear associations, between categorical and scale variables. The level of significance was set at 0.05 .

\section{RESULTS}

The review results show that the analysed works try to understand the reasons for low patients' adherence to eHealth technologies $(n=10 ; 77 \%)$, resulting that privacy and trust can influence the continued use of applications $(n=10 ; 77 \%)$. The sample includes articles from: 2020=2;2019=8; $2018=1 ; 2017=1$; and $2016=1$. 
Table 1. Results from the literature review of published works for the past 5 years

\begin{tabular}{llcc}
\hline $\begin{array}{l}\text { Database } \\
\text { Engines }\end{array}$ & \multicolumn{1}{c}{ Queries } & $\begin{array}{c}\text { Before } \\
\text { titles/ } \\
\text { abstracts }\end{array}$ & $\begin{array}{c}\text { After } \\
\text { titles/ } \\
\text { abstracts }\end{array}$ \\
\hline SCOPUS & $\begin{array}{l}\text { TITLE-ABS-KEY ((health OR disease or chronic) AND (privacy OR trust) } \\
\text { AND (perception OR attitude) AND (mhealth OR mobile OR app)) }\end{array}$ & 186 & 4 \\
\hline PUBMED & $\begin{array}{l}\text { (health OR disease OR chronic) AND ("privacy" OR "trust") AND } \\
\text { ("perception" OR "attitude") AND (mhealth OR mobile OR app)) }\end{array}$ & 160 & 1 \\
\hline ISI WOK & $\begin{array}{l}\text { TS=((health OR disease OR Chronic) AND ("privacy" OR “TRUST") } \\
\text { AND ("perception" OR "attitude") AND (mhealth OR mobile OR app)) }\end{array}$ & 49 & 8 \\
\hline TOTAL & & $\mathbf{3 9 5}$ & $\mathbf{1 3}$ \\
\hline
\end{tabular}

A total of 66 participants answered the complete survey with the security questions. The mean age of the sample who responded the security questions is 27.7 years $(\mathrm{N}=65)$, with a standard deviation of 12.9 and the youngest participant being 13 years old and the oldest 70 . The majority of respondents were women $(59 \%)$, with secondary education (70\%), 48\% were students, 39\% employed and 9\% unemployed. Also, 98\% of respondents use social networks, with $66 \%$ all the time or several times a day $(89 \%$ Facebook; 38\% Instagram; $51 \%$ another network). Moreover, $75 \%$ of participants use smartphones and $23 \%$ use tablets or iPad, to: Access GPS (32\% never or once a month); Browse the Internet (36\% all the time and $42 \%$ several times a day); Take pictures (16\% several times a day and $27 \%$ sometimes per week); Use mobile applications (63\% all the time or several times per day); and Play games (30\% several times per day or per week and $27 \%$ never).

In addition, participants have already downloaded and used health and fitness applications (50\%) but only a minority did the same for asthma applications (1\%). However, $89 \%$ of participants would like to use an asthma application. Table 2 describes the answers to the four security questions and the median of the "health status score" (0-100) they gave themselves on the day they were enquired, in relation to the security answers. Tables 3 and 4 present the results from the application of Spearman and Eta statistical tests, respectively, the authors believe can be analysed in future research.

Table 2. Answers to the four security questions by the participants $(\mathrm{N}=66)$ with health status' score median $(\mathrm{Hsm})$

\begin{tabular}{|c|c|c|c|c|c|c|c|}
\hline & \multicolumn{2}{|c|}{ NEVER } & \multicolumn{2}{|c|}{ SOMETIMES } & \multicolumn{2}{|c|}{ ALWAYS } & Don't know \\
\hline Security questions & n (\%) & Hsm & n (\%) & Hsm & n (\%) & Hsm & $\mathbf{n}$ \\
\hline Lets app access to the location & $24(36)$ & 84 & $37(56)$ & 85 & $5(8)$ & 85 & 0 \\
\hline Lets app access photographs & $34(51)$ & 85 & $28(42)$ & 84 & $3(5)$ & 60 & 1 \\
\hline Lets app send notifications & $13(20)$ & 90 & $31(47)$ & 83 & $20(30)$ & 81 & 2 \\
\hline Lets app access the contacts & $46(70)$ & 83 & $19(29)$ & 87 & $0(0)$ & 0 & 1 \\
\hline
\end{tabular}

Table 3. Relations and statistical significance found in the sample using Spearman rank correlation test

\begin{tabular}{lccc}
\hline Demographics and health & Security variables & Value & $\begin{array}{c}\text { Sig. } \\
\text { (2-tailed) }\end{array}$ \\
\hline $\begin{array}{l}\text { Last week: felt very anxious } \\
\text { with fear and a tight stomach }\end{array}$ & Contacts & .245 & $\mathbf{. 0 4 7}$ \\
Education level & Photographs & -.358 & $\mathbf{. 0 0 3}$ \\
\hline $\begin{array}{l}\text { Last week: felt fear as if something } \\
\text { terrible is going to happen }\end{array}$ & Contacts & .228 & $.066 \mathrm{~ns}$ \\
\hline Last week: felt restless and cannot stop & Notifications & -.221 & $.074 \mathrm{~ns}$ \\
\hline Last week: felt tense or nervous & Notifications & -.220 & $.076 \mathrm{~ns}$ \\
\hline Pain/Feeling bad & Photographs & -.215 & $.083 \mathrm{~ns}$ \\
\hline
\end{tabular}

In bold, the statistically significant associations $(p<.05)$; ns=not-significant $(p>.05)$

Moreover, each one of the continuous variables "health status score" and "adherence to inhaler" was tested for association with the security variables (Table 4).

Table 4. Eta coefficient test for security (dependent), "health status" and "adherence to inhaler" (independent) variables

\begin{tabular}{lcccc}
\hline Independent/Dependent & Location & Photographs & Notifications & Contacts \\
\hline Health status & .477 & .531 & .349 & .571 \\
\hline Adherence to inhaler & .619 & $\mathbf{. 6 5 2}$ & $\mathbf{. 6 9 2}$ & .613 \\
\hline
\end{tabular}

In bold, $\mathrm{Eta}^{2}$ value with moderate to strong association $\left(\mathrm{Eta}^{2}\right.$ value $>.7$ is a strong association) 


\section{DISCUSSION}

In general, participants answer the options "Never" or "Sometimes" to the four security alternatives, so they appear to be a moderate/high IT literate and privacy-aware sample, because the most permissive answers are related to the application sending "Notifications", with the highest number of "Always", and the least permissive is accessing the "Contacts". However, although the participants chose to block most accesses to applications when generically asked, they allowed access to those features to the specific InspirerMundi application, to participate in the study. This underlines the idea that the goal, context and needs relating to their well-being can influence their privacy perception. Still, a connection between privacy awareness and good, to very good, health status, may be worth further testing, with a more balanced and larger sample. Also, most "Sometimes" selections are for letting access to "Location" and send "Notifications" and the most "Never" selections are for letting access to "Photographs" and "Contacts", maybe because the later are considered to be closer to private matters, and location is not yet a concept for people to closely link with their privacy. With the current pandemic and the increase use of contact tracing applications, this perception may change overtime.

In terms of statistically significant findings (Table 3), the only correlation between patients' well-being and their security options is between "Feeling very anxious and with fear", which positively correlates with "Contacts". This needs further clarification as "Contacts" have more "Never" selections, from the four options.

Although not directly related to healthcare, the other statistically significant finding relates to the "Education level", which is negatively correlated with "Photographs" ( $p$-value<.001). If this can be confirmed, then different types of personal data, context and experience may affect different types of privacy perception.

While all the other associations are not statistically significant, it may be worth noting relations that have a $p$-value close to .05 , such as: i) the "Feeling something terrible is going to happen" is positively correlated with "Contacts" (which corroborates the statistically significant relation already described in Table 3, $1^{\text {st }}$ row); ii) the "Feeling of restlessness and unable to stop" is negatively correlated with "Notifications"; and iii) the "Feeling pain/bad" is negatively correlated with "Photographs". These need to be verified with a larger sample.

In terms of relations between the "health status score" and "adherence to inhaler" variables, with the security variables (Table 4), stronger associations were found between the later and the security variables, than with the "health status score" variable. And from these stronger associations, the strongest relate to "Notifications" and "Photographs", which may be explained by the specificities of the tested application, which includes notifications to remind patients to take medication and images to register inhaler medication intake. For the "health status score", there are two moderate associations with the two variables "Photographs" (corroborating with results from last row, Table 3, where people with "Pain/feeling bad" associate negatively with "Photographs") and "Contacts", with much less association with "Notifications".

Other particular relations that may require some attention are with participants: 1) that "Never sit or relax, or feel some pain" choose the highest number of "Never"; 2) who are "Not in pain" give many times access to "Photographs" (already discussed); 3) with regular "Good mobility", give "Sometimes" accesses to all data, except for the "Contacts"; and 4) participants with "More interest in their appearance" contradict most of the analysed sample and are the ones that give more "Sometimes" accesses to "Contacts".

Limitations: the small sample size; the self-reported health status score of the analysed sample which is, in general, good to very good, and does not provide a balanced sample in terms of well-being to offer a wider range of comparisons and associations; the literature review was performed by only one reviewer; and, due to space constraints, a more detailed presentation of results and discussion was not possible.

\section{CONCLUSION}

This preliminary study focuses on understanding if there is a link between patients' health outcomes and their perceptions of privacy and trust in mhealth applications. Many questions were raised that need further investigation; however, findings show that privacy perception may change according to demographic factors or type of personal data being used/accessed together with context and patients' well-being. Results motivate the authors to proceed research in this area because, if strong links are found between well-being or health related outcomes and privacy related data or actions, providing mobile applications with features that can nudge chronic disease patients to improve adherence to medication and therapeutics, and be more confident to trust those applications, can become a possibility. 


\section{ACKNOWLEDGEMENT}

This work has been funded by TagUBig - Taming Your Big Data (IF/00693/2015) from Researcher FCT Program funded by National Funds through FCT; and has been supported by ERDF (European Regional Development Fund) through the operations: POCI-01-0145-FEDER-029130 (mINSPIRERS - mHealth to measure and improve adherence to medication in chronic obstructive respiratory diseases - generalisation and evaluation of gamification, peer support and advanced image processing technologies) cofunded by the COMPETE2020 (Programa Operacional Competitividade e Internacionalização), Portugal 2020 and by Portuguese Funds through FCT (Fundação para a Ciência e a Tecnologia).

\section{REFERENCES}

Birkhäuer, J., Gaab, J., Kossowsky, J., Hasler, S., Krummenacher, P., Werner C, et al, 2017. Trust in the health care professional and health outcome: A meta-analysis. In PLoS ONE, 12(2): e0170988.

Ferreira, A., Almeida, R., Jácome, C., Fernandes, J., Fonseca, J.A. and Vieira-Marques, P., 2019. How inspiring is your app? A usability take on an app for asthma medication adherence. Proceedings of the International Conference on e-Health, EH 2019, pp. 225-229.

Hong, Z., Deng, Z. and Zhang, W., 2018. Examining factors affecting patients trust in online healthcare services in China: The moderating role of the purpose of use. In Health Informatics Journal, 25.

Jácome, C., Almeida, R., Teixeira, J.P., Vieira-Marques, P., Vilaça, R., Fernandes, J.M., Ferreira, A. and Fonseca, J.A., 2017. Inspirers: an app to measure and improve adherence to inhaled treatment. Proceedings of the International Conference on E-Health, EH 2017, 135-139.

Jácome, C., et al., 2018. mINSPIRERS - Feasibility of a mobile application to measure and improve adherence to inhaled controller medications among adolescents and adults with persistent asthma: Protocol for a multicentre observational study. In Rev Port Imunoalergologia, 26:1 47-61.

Kruse, C., Betancourt, J., Ortiz, S., Valdes Luna, SM., Bamrah, IK and Segovia, N., 2019. Barriers to the Use of Mobile Health in Improving Health Outcomes in Developing Countries: Systematic Review. In J Med Internet Res, 21(10):e13263.

Larson, RS., 2018. A Path to Better-Quality mHealth Apps. In JMIR Mhealth Uhealth, 6(7):e10414.

Majid, H., Jetzabel, S. and Kai, R., 2019. Revealing the unrevealed: Mining smartphone users privacy perception on app markets. In Computers \& Security, Volume 83, Pages 332-353.

Polinski JM., Kesselheim, AS., Frolkis, JP., Wescott, P., Allen-Coleman, C. and Fischer, MA., 2014. A matter of trust: patient barriers to primary medication adherence. In Health Education Research, Volume 29, Issue 5, Pages 755-763.

Saheb, T., 2020. An empirical investigation of the adoption of mobile health applications: integrating big data and social media services. In Health Technol. (2020).

Serenko, N. and Fan, L., 2013. Patients' perceptions of privacy and their outcomes in healthcare. In Int. J. Behavioural and Healthcare Research, Vol. 4, No. 2.

van Rooy, D. and Bus, J, 2010. Trust and privacy in the future internet—a research perspective. In IDIS 3, 397-404.

Vervier, L., Valdez, A. and Ziefle, M., 2018. "Should I Trust or Should I Go?” or What Makes Health-Related Websites Appear Trustworthy? - An Empirical Approach of Perceived Credibility of Digital Health Information and the Impact of User Diversity. Proceedings of the 4th International Conference on Information and Communication Technologies for Ageing Well and e-Health (ICT4AWE 2018), pages 169-177.

Vo, V., Auroy, L. and Sarradon-Eck, A., 2019. Patients' Perceptions of mHealth Apps: Meta-Ethnographic Review of Qualitative Studies. JMIR Mhealth Uhealth, 7(7):e13817.

Wei, J., Vinnikova, A., Lu, L. and Xu, J., 2020. Understanding and Predicting the Adoption of Fitness Mobile Apps: Evidence from China [published online ahead of print, 2020 Feb 10]. In Health Commun, 1-12.

Woldeyohannes, H.O. and Ngwenyama, O.K., 2017. Factors Influencing Acceptance and Continued Use of mHealth Apps. In: Nah FH., Tan CH. (eds) HCI in Business, Government and Organizations. Interacting with Information Systems. HCIBGO. Lecture Notes in Computer Science, vol 10293. Springer, Cham.

Zhou, L., Bao, J., Watzlaf, V. and Parmanto, B., 2019. Barriers to and Facilitators of the Use of Mobile Health Apps from a Security Perspective: Mixed-Methods Study. In JMIR Mhealth Uhealth, 7(4):e11223. 